\title{
Model of Dirac field interacting with material plane within Symanzik's approach
}

\author{
Yu. M. Pismak ${ }^{1, a}$ and D. Yu. Shukhobodskaia ${ }^{1}$ \\ ${ }^{1}$ Saint-Petersburg State University
}

\begin{abstract}
The model for the interaction of a spinor field with a material plane is constructed in the framework of the Symanzik's approach. The characteristics of scattering process of Dirac particles on the plane are calculated. The bounced states localized near the plane are investigated.The model can find application to a wide class of phenomena arising by the interaction of quantum electrodynamics fields with two-dimensional materials.
\end{abstract}

\section{Introduction}

Space-time homogeneity and isotropy are typical for usual quantum field theory models of elementary particles [1]. It is a natural assumption in the study of various processes with simplest excitations of quantum vacuum. However, if the properties of the vacuum change essentially as a result of the interaction of quantum fields with macroscopic objects, then such an approach is inapplicable. In this case, quantum macro-effects may appear in dynamics of material bodies which cannot be explained in the framework of classical physics.

Theoretically, this problem was first considered in 1948 by Casimir [2]. He showed that as the result of fluctuations of the quantum vacuum, attraction occurs between two ideally conducting plates of a planar uncharged capacitor. This phenomenon, called the Casimir effect (CE), is observed experimentally [3-6], and the empirical results obtained for materials with high conductivity are, with a high degree of accuracy, in agreement with theoretical ones.

At typical for CE distances of 10 to $1000 \mathrm{~nm}$ both classical and quantum properties of the system turn out to be essential. Their combination forms a special nanophysics. Investigation of it is not solely of theoretical interest, understanding its regularities is also important for developing new technological devices, in view of the increasing trend to their miniaturization.

Although theoretical investigations of the $\mathrm{CE}$ are the subject of numerous works [6,7], they are often based on simplified models of a free scalar field theory or free electromagnetic field with fixed boundary conditions, applying only to investigations of some particular aspect of the $\mathrm{CE}$, and ignoring usually specificity of quantum electrodynamics. Such models are not suitable for comprehensively describing a wide range of nanophysical phenomena occurring in a system as a result of the interaction of its quantum degrees of freedom with material bodies of a given shape (classic defect).

ae-mail: ypismak@yahoo.com 
The results presented in our paper were obtained within the Symanzik's approach [8], in which the interaction of quantized fields with a spatial inhomogeneity is modeled by an additional action functional (defect action), concentrated in the spatial domain where this inhomogeneity - a macroscopic object - is located. An important assumption is also that the standard requirements for quantum field models (locality, renormalizability, symmetry properties) are fulfilled.

Special attention is given to quantum field models with two-dimensional spatial inhomogeneities due to the recent progress in the physics of two-dimensional material. In quantum electrodynamics (QED), the interaction of photons with a two-dimensional defect is entirely determined by the requirements of locality, gauge invariance, and renormalizability and is described by a Chern-Simons action functional with one dimensionless constant characterizing the properties of the material [9]. The Casimir force depends on that constant and thus become nonuniversal. For a planar capacitor it can be not only attractive but also repulsive [9]. The interaction of electromagnetic waves with two-dimensional planar defects in the model with a Chern-Simons potential was investigated in [10] - [12].

In this paper we use Symanzik's approach in the standard QED in (3+1)-dimensional space-time to model the interaction of a two-dimensional material object with the Dirac field. Such a problem for a spinor field was considered in [13]. In the defect action functional, in addition to the photon Chern-Simons action, a purely Dirac contribution must be taken into account in accordance with the basic principles of QED. In this model framework, we investigate scattering of spinor particles on a material plane and also consider bound states localized in its vicinity.

\section{Statement of problem}

The proposed by Symanzik action functional describing the interaction of the quantum field with material object (defect) has the form:

$$
S(\varphi)=S_{V}(\varphi)+S_{d e f}(\varphi)
$$

where $S_{V}$ is the action of the original quantum field system and $S_{d e f}$ is the defect action:

$$
S_{V}(\varphi)=\int L(\varphi(x)) d^{D} x, S_{d e f}(\varphi)=\int_{\Gamma} L_{d e f}(\varphi(x)) d^{D^{\prime}} x
$$

Here $\Gamma$ is a subspace of dimension $D^{\prime} \leq D$ in a D-dimensional space [8]. The basic principles of QEDgauge invariance, locality, and renormalizability-impose strong constraints on the possible form of the defect action $S_{d e f}$. It follows that interaction of the photon field $A_{\mu}(x)$ with a two-dimensional surface without charges and currents and shape defined by equation $\Phi(x)=0, x=\left(x_{0}, x_{1}, x_{2}, x_{3}\right)$, is described by the Chern-Simons functional

$$
S_{d e f}(A)=\frac{a}{2} \int \varepsilon^{\lambda \mu v \rho} \partial_{\lambda} \Phi(x) A_{\mu}(x) F_{v \rho}(x) \delta(\Phi(x)) d^{4} x,
$$

where $F_{\nu \rho}(x)=\partial_{v} A_{\rho}-\partial_{\rho} A_{v}, \varepsilon^{\lambda \mu \nu \rho}$ is the totally antisymmetric tensor $\left(\varepsilon^{0123}=1\right)$, and the parameter $a$ is a dimensionless coupling constant. This expression is the most general form of a gauge-invariant action concentrated on a defect surface being invariant in respect to reparameterization of one and not having any negative-dimension parameters. A fermionic contribution to the defect action in the most general form is written as

$$
S_{d e f}(\bar{\psi}, \psi)=\sum_{j=1}^{16} \int \alpha_{j} \bar{\psi}(x) \Gamma_{j} \psi(x) \delta(\Phi(x)) d x,
$$


where $\Gamma_{j}$ are the 16 basis Dirac matrices and $\alpha_{j}$ are dimensionless coupling constants. The full action of the model, which satisfies the basic principles of QED, has the form

$$
S(\bar{\psi}, \psi, A)=-\frac{1}{4} F_{\mu \nu} F^{\mu \nu}+\bar{\psi}(i \hat{\partial}-m+i e \hat{A}) \psi+S_{d e f}(A)+S_{d e f}(\bar{\psi}, \psi) .
$$

By virtue of the renormalizability requirement, the coupling of fields is described by the standard term $i e \bar{\psi} \hat{A} \psi$ in the QED action.

In this paper we consider the defect, which lie on the material plane $x_{3}=0$. Then in the Dirac part of the action of our model,

$$
S(\bar{\psi}, \psi)=\int \bar{\psi}(x)\left(i \hat{\partial}-m+\Omega\left(x_{3}\right)\right) \psi(x) d x,
$$

the coupling of the spinor field to the plane is described by the matrix $\Omega\left(x_{3}\right)=Q \delta\left(x_{3}\right)$. Because $\Omega\left(x_{3}\right)$ and $\delta\left(x_{3}\right)$ have the dimension of mass, the matrix $Q$ is dimensionless. By the symmetry of the system, it must be invariant under transformations in the subgroup of the Poincare group not changing the $x_{3}$ coordinate. In the most general case, the matrix $Q$ can be represented as

$$
Q=q_{1} I+q_{2} \gamma_{5}+q_{3} \gamma_{3}+q_{4} \gamma_{5} \gamma_{3}
$$

where $I$ is the unit $4 x 4$ matrix and $\gamma_{3}$ and $\gamma_{5}=i \gamma_{0} \gamma_{1} \gamma_{2} \gamma_{3}$ are Dirac matrices, for which we use the representation

$$
\begin{gathered}
\gamma_{0}=\left(\begin{array}{cccc}
1 & 0 & 0 & 0 \\
0 & 1 & 0 & 0 \\
0 & 0 & -1 & 0 \\
0 & 0 & 0 & -1
\end{array}\right), \gamma_{1}=\left(\begin{array}{cccc}
0 & 0 & 0 & 1 \\
0 & 0 & 1 & 0 \\
0 & -1 & 0 & 0 \\
-1 & 0 & 0 & 0
\end{array}\right), \\
\gamma_{2}=\left(\begin{array}{cccc}
0 & 0 & 0 & -i \\
0 & 0 & i & 0 \\
0 & i & 0 & 0 \\
-i & 0 & 0 & 0
\end{array}\right), \gamma_{3}=\left(\begin{array}{cccc}
0 & 0 & 1 & 0 \\
0 & 0 & 0 & -1 \\
-1 & 0 & 0 & 0 \\
0 & 1 & 0 & 0
\end{array}\right) .
\end{gathered}
$$

The motion of a spinor particle in the field of the defect $\Omega\left(x_{3}\right)$ is described by the Dirac equation

$$
\left(i \hat{\partial}-m+\Omega\left(x_{3}\right)\right) \psi(x)=0 .
$$

It is one of the Euler-Lagrange equations for action (1), obtained by taking the functional derivative of the action with respect to $\bar{\psi}(x)$. Taking the derivative of (1) with respect to $\psi(x)$ yields the second equation:

$$
\left(-i \partial_{\mu} \bar{\psi}(x)\right) \gamma^{\mu}+\bar{\psi}(x)\left(m-\Omega\left(x_{3}\right)\right)=0 .
$$

It can be easily verified that this equation can be obtained for $\bar{\psi}(x)=\psi^{*}(x) \gamma_{0}$ by the Dirac conjugation of equation (3), if $\gamma_{0} \Omega^{+}\left(x_{3}\right)=\Omega\left(x_{3}\right) \gamma_{0}$.

We introduce the following notation

$$
\bar{x}=\left(x_{0}, x_{1}, x_{2}\right), \overline{p x}=p_{0} x_{0}-p_{1} x_{1}-p_{2} x_{2} .
$$

for (2+1)- parts of space-time vectors and represent $\psi(x)=\psi\left(\bar{x}, x_{3}\right)$ as $\psi(x)=\psi_{s}(x)+\psi_{a}(x)$, where

$$
\psi_{s}(x)=\frac{1}{2}\left(\psi\left(\bar{x}, x_{3}\right)+\psi\left(\bar{x},-x_{3}\right)\right), \psi_{a}(x)=\frac{1}{2}\left(\psi\left(\bar{x}, x_{3}\right)-\psi\left(\bar{x},-x_{3}\right)\right) .
$$


Analysis of the possible singularities of spinor $\psi(x)$ at the point $x_{3}=0$, which was made in the paper [14], leads to the following: for $x_{3} \neq 0$, the field $\psi(x)$ satisfies the Dirac equation

$$
(i \hat{\partial}-m) \psi(x)=0,
$$

and the relation

$$
-i \gamma_{3} \psi_{a}(\bar{x})+Q \psi_{s}(\bar{x})=0
$$

holds at $x_{3}=0$. The general solution of (4) with $x_{3}>0$ can be represented as

$$
\psi(x)=\frac{1}{(2 \pi)^{3}} \int e^{i \bar{p} \bar{x}} \psi_{+}\left(\bar{p}, x_{3}\right) d \bar{p} .
$$

Then it follows from (4) that $\psi_{+}\left(\bar{p}, x_{3}\right)$ satisfies the equation

$$
\left(i \gamma_{3} \partial_{3}+\hat{\bar{p}}+m\right) \psi_{+}\left(\bar{p}, x_{3}\right)=0 .
$$

Its general solution has the form

$$
\psi_{+}\left(\bar{p}, x_{3}\right)=U\left(\bar{p}, x_{3}\right) \chi_{+}(\bar{p}), U\left(\bar{p}, x_{3}\right)=e^{-i \gamma_{3}(\hat{p}+m) x_{3}} .
$$

where $\chi_{+}(\bar{p})$ is an arbitrary spinor depending only on $\bar{p}$.

Similarly, for $x_{3}<0$, we obtain

$$
\psi(x)=\frac{1}{(2 \pi)^{3}} \int e^{i \bar{p} \bar{x}} \psi_{-}\left(\bar{p}, x_{3}\right) d \bar{p}, \psi_{-}\left(\bar{p}, x_{3}\right)=e^{-i \gamma_{3}(\hat{\bar{p}}+m) x_{3}} \chi_{-}(\bar{p}) .
$$

From (5), (6), (7) we obtain the relation

$$
-i \gamma_{3}\left(\chi_{+}-\chi_{-}\right)+Q\left(\chi_{+}+\chi_{-}\right)=0
$$

which implies that

$$
\chi_{-}=S \chi_{+}, S=\left(i \gamma_{3}+Q\right)^{-1}\left(i \gamma_{3}-Q\right) .
$$

We introduce the notation

$$
\kappa(\bar{p})=\sqrt{\bar{p}^{2}-m^{2}}, P^{ \pm}(\bar{p})=\frac{1}{2}\left(1 \pm \frac{\gamma_{3}(\hat{\bar{p}}+m)}{\kappa(\bar{p})}\right) .
$$

We can then represent $U\left(\bar{p}, x_{3}\right)$ as

$$
\begin{array}{r}
U\left(\bar{p}, x_{3}\right)=e^{-i \gamma_{3}(\hat{p}+m) x_{3}}=\cos \left(\kappa(\bar{p}) x_{3}\right)-i \sin \left(\kappa(\bar{p}) x_{3}\right) \frac{\gamma_{3}(\hat{\bar{p}}+m)}{\kappa(\bar{p})}= \\
=e^{-i \kappa(\bar{p}) x_{3}} P^{+}(\bar{p})+e^{i \kappa(\bar{p}) x_{3}} P^{-}(\bar{p}) .
\end{array}
$$

The matrices $P^{ \pm}(\bar{p})$ are projectors:

$$
\begin{array}{r}
P^{ \pm}(\bar{p}) P^{ \pm}(\bar{p})=P^{ \pm}(\bar{p}), P^{+}(\bar{p})+P^{-}(\bar{p})=1, \\
P^{+}(\bar{p}) P^{-}(\bar{p})=P^{-}(\bar{p}) P^{+}(\bar{p})=0 .
\end{array}
$$

Each of them has two two-dimensional eigensubspaces $L^{+}$and $L^{-}$, which are represented as linear combinations of two mutually orthogonal eigenvectors $v_{1}^{ \pm}$and $v_{2}^{ \pm}$:

$$
\begin{array}{r}
L^{ \pm}\left(a_{1}^{ \pm}, a_{2}^{ \pm}\right)=a_{1}^{ \pm} v_{1}^{ \pm}+a_{2}^{ \pm} v_{2}^{ \pm}, \\
P^{ \pm}(\bar{p}) L^{ \pm}\left(a_{1}^{ \pm}, a_{2}^{ \pm}\right)=L^{ \pm}\left(a_{1}^{ \pm}, a_{2}^{ \pm}\right), P^{ \pm}(\bar{p}) L^{\mp}\left(a_{1}^{\mp}, a_{2}^{\mp}\right)=0 .
\end{array}
$$


Here, $a_{i}^{ \pm}, i=1,2$, are complex parameters, and $v_{i}^{ \pm}, i=1,2$, are spinors, which we choose as

$$
\begin{array}{r}
v_{1}^{+}=n_{1}^{-1}\left(-h_{1}, h_{2}^{-}, 1, h_{3}^{-}\right), v_{2}^{+}=n_{2}^{-1}\left(h_{1}, h_{2}^{+}, 1, h_{3}^{+}\right), \\
v_{1}^{-}=n_{2}^{-1}\left(-h_{1}, h_{2}^{+}, 1,-h_{3}^{+}\right), v_{2}^{-}=n_{1}^{-1}\left(h_{1}, h_{2}^{-}, 1,-h_{3}^{-}\right) \\
n_{1}=2 \sqrt{\frac{p_{0} h_{2}^{-}}{\left(p_{1}+i p_{2}\right)}}, n_{2}=2 \sqrt{\frac{p_{0} h_{2}^{+}}{\left(p_{1}+i p_{2}\right)}}, h_{1}=\frac{\sqrt{p_{0}^{2}-m^{2}}}{m+p_{0}}, \\
h_{2}^{ \pm}=\frac{p_{0}^{2}-m^{2} \pm \sqrt{p_{0}^{2}-m^{2}} \kappa(\bar{p})}{\left(m+p_{0}\right)\left(p_{1}-i p_{2}\right)}, h_{3}^{ \pm}=\frac{\kappa(\bar{p}) \pm \sqrt{p_{0}^{2}-m^{2}}}{p_{1}-i p_{2}} .
\end{array}
$$

These spinors are eigenstates of the helicity operator

$$
\sigma(\vec{p})=\frac{i}{2|\vec{p}|}(\vec{p} \vec{s}), \vec{s}=\left(\gamma_{2} \gamma_{3},-\gamma_{1} \gamma_{3}, \gamma_{1} \gamma_{2}\right)
$$

They satisfy the relations

$$
\left.\sigma(\vec{p})\right|_{p_{3}=\mp \kappa(\bar{p})} v_{1}^{ \pm}=-\frac{1}{2} v_{1}^{ \pm},\left.\sigma(\vec{p})\right|_{p_{3}=\mp \kappa(\bar{p})} v_{2}^{ \pm}=\frac{1}{2} v_{2}^{ \pm}
$$

We represent the spinors $\chi_{ \pm}(\bar{p})$ as

$$
\chi_{ \pm}(\bar{p})=b_{ \pm}^{1}(\bar{p}) v_{1}^{-}(\bar{p})+b_{ \pm}^{2}(\bar{p}) v_{2}^{-}(\bar{p})+c_{ \pm}^{1}(\bar{p}) v_{1}^{+}(\bar{p})+c_{ \pm}^{2}(\bar{p}) v_{2}^{+}(\bar{p})
$$

The equality

$$
\chi_{ \pm}^{*}(\bar{p}) \gamma_{0} \gamma_{3} \chi_{ \pm}(\bar{p})=\bar{\chi}_{ \pm}(\bar{p}) \gamma_{3} \chi_{ \pm}(\bar{p})=\frac{\kappa(\bar{p})}{p_{0}}\left(\left|c_{ \pm}^{1}\right|^{2}+\left|c_{ \pm}^{2}\right|^{2}-\left|b_{ \pm}^{1}\right|^{2}-\left|b_{ \pm}^{2}\right|^{2}\right)
$$

can be easily verified. Hence, if $p_{0}>0$ and $\kappa(\bar{p})>0$, then the amplitudes $c_{ \pm}^{i}$ describe the motion of particles in the direction $x_{3}>0$, and $b_{ \pm}^{i}$ describe the motion in the opposite direction.

Based on Eqs. (3), we investigate the scattering of spinor particles on the defect plane and also the properties of bound states localized in the vicinity of the defect.

\section{Scattering of particles on the plane $x_{3}=0$}

Let us consider the process where the initial flow of particles $I_{\text {in }}$ with the momentum $\vec{p}=p_{1}, p_{2}, \kappa(\bar{p})$ moves toward the plane $x_{3}=0$ from the domain $x_{3}<0$. As it was mentioned above, if $p_{0}>0$, then the amplitudes $c_{ \pm}^{1}$ and $c_{ \pm}^{2}$ describe particles moving in the positive direction of the $x_{3}$ axis, and $b_{ \pm}^{1}$ and $b_{ \pm}^{2}$ describe particles moving in the negative direction. Hence the properties of the scattering process are determined by the amplitudes $c_{-}^{1}$ and $c_{-}^{2}$. In the domain $x_{3}<0$, there is also the flux of reflected particles specified by the amplitudes $b_{-}^{1}$ and $b_{-}^{2}$. In the domain $x_{3}>0$, there are only particles moving in the positive direction. Their flux is described by the amplitudes $c_{+}^{1}$ and $c_{+}^{2}$. Hence, for the considered process, the reflection and transmission coefficients $K_{r}$ and $K_{t r}$ have the forms

$$
K_{r}=\frac{\left|b_{-}^{1}\right|^{2}+\left|b_{-}^{2}\right|^{2}}{\left|c_{-}^{1}\right|^{2}+\left|c_{-}^{2}\right|^{2}}, \quad K_{t r}=\frac{\left|c_{+}^{1}\right|^{2}+\left|c_{+}^{2}\right|^{2}}{\left|c_{-}^{1}\right|^{2}+\left|c_{-}^{2}\right|^{2}} .
$$

The amplitudes $c_{-}^{1}, b_{-}^{1}$ and $c_{+}^{1}$ correspond to particles with negative helicity, and $c_{-}^{2}, b_{-}^{2}$, and $c_{+}^{2}$, to those with positive helicity. For the scattering process considered here, the amplitudes $c_{-}^{1}$ and $c_{-}^{2}$ are 
considered fixed, and $b_{-}^{1}, b_{-}^{2}, c_{+}^{1}$, and $c_{+}^{2}$ are determined from Eq. (8), which relates $\chi_{+}$to $\chi_{-}$, and from the equations

$$
b_{+}^{1}=0, b_{+}^{2}=0
$$

From analysis of Eq. (8), which is necessary for solving our problem, it could be obtained, that choosing the parameters $\varsigma_{1}, \varsigma_{2}, \varsigma_{3}$, and $\varsigma_{4}$ to be real, we can represent the matrix $S$ as $S=e^{i \varphi} \mathcal{S}$, where $0 \leq \varphi<2 \pi$ and

$$
\begin{array}{r}
\mathcal{S}=\varsigma_{1} I+i \varsigma_{2} \gamma_{5}+i \varsigma_{3} \gamma_{3}+\varsigma_{4} \gamma_{5} \gamma_{3}, \\
\varsigma_{1}^{2}+\varsigma_{2}^{2}-\varsigma_{3}^{2}-\varsigma_{4}^{2}=1 .
\end{array}
$$

From (8), $Q$ can be expressed in terms of $S$ :

$$
Q=i \gamma_{3}(1-S)(1+S)^{-1} \text {. }
$$

Hence, with (12), we express the coefficients $q_{i}, 1 \leq i \leq 4$, in formula (2) in terms of the parameters $\varsigma_{i}$ :

$$
q_{1}=-\frac{\varsigma_{3}}{1+\varsigma_{1}}, q_{2}=-\frac{i \varsigma_{4}}{1+\varsigma_{1}}, q_{3}=0, q_{4}=-\frac{\varsigma_{2}}{1+\varsigma_{1}} .
$$

It follows from these relations that $q_{2}$ is imaginary and $q_{1}$ and $q_{4}$ are real. Relations (12) and (13) allow expressing $\varsigma_{i}$ in terms of $q_{i}$ :

$$
\begin{array}{r}
\varsigma_{1}=-1+\frac{2}{1-q_{1}^{2}+q_{2}^{2}+q_{4}^{2}}, \varsigma_{2}=-\frac{2 q_{4}}{1-q_{1}^{2}+q_{2}^{2}+q_{4}^{2}}, \\
\varsigma_{3}=-\frac{2 q_{1}}{1-q_{1}^{2}+q_{2}^{2}+q_{4}^{2}}, \varsigma_{4}=\frac{2 i q_{2}}{1-q_{1}^{2}+q_{2}^{2}+q_{4}^{2}} .
\end{array}
$$

Substituting the expression for the matrix $S$ of form (12) and $\chi_{ \pm}$of form (9) in Eq. (8), we obtain linear equations for the amplitudes $b_{ \pm}^{1}, b_{ \pm}^{2}, c_{+}^{1}$, and $c_{+}^{2}$. Their solution with Eq. (11) taken into account allows finding the fluxes of the reflected and transmitted particles of different polarizations. For compact writing the obtained results we use the following notations

$$
\omega=\sqrt{p_{0}^{2}-m^{2}}, \kappa=\sqrt{p_{0}^{2}-m^{2}-p_{1}^{2}-p_{2}^{2}}
$$

The reflection and transmission amplitudes of considered scattering process read

$$
\begin{array}{r}
b_{-}^{1}=\frac{\left(c_{-}^{1} m\left(m \varsigma_{33-22}+i \kappa \varsigma_{13+24}\right) \sqrt{\omega^{2}-\kappa^{2}}+c_{-}^{2} \kappa\left(\kappa\left(\omega \varsigma_{14-23}-i p_{0} \varsigma_{13+24}\right)+m\left(p_{0} \varsigma_{22-33}+i \varsigma_{12-34} \omega\right)\right)\right)}{g}, \\
b_{-}^{2}=\frac{\left(c_{-}^{2} m\left(m \varsigma_{33-22}+i \kappa \varsigma_{13+24}\right) \sqrt{\omega^{2}-\kappa^{2}}+c_{-}^{1} \kappa\left(\kappa\left(\omega \varsigma_{14-23}+i p_{0} \varsigma_{13+24}\right)+m\left(p_{0} \varsigma_{33-22}+i \omega \varsigma_{12-34}\right)\right)\right)}{g}, \\
c_{+}^{1}=\frac{\kappa\left(c_{-}^{1}\left(\kappa\left(\omega \varsigma_{1}+i p_{0} \varsigma_{2}\right)-i m \varsigma_{3} \omega\right)+i c_{-}^{2} m \varsigma_{2} \sqrt{\omega^{2}-\kappa^{2}}\right)}{g}, \\
c_{+}^{2}=\frac{\kappa\left(c_{-}^{2}\left(\kappa\left(\omega \varsigma_{1}-i p_{0} \varsigma_{2}\right)-i m \varsigma_{3} \omega\right)+i c_{-}^{1} m \varsigma_{2} \sqrt{\omega^{2}-z^{2}}\right)}{g},
\end{array}
$$

where

$$
g=\omega\left(\left(m^{2}+\kappa^{2}\right) \varsigma_{2}^{2}+\left(\kappa \varsigma_{1}-i m \varsigma_{3}\right)^{2}\right), \quad \varsigma_{i k}=\varsigma_{i} \varsigma_{k}, \quad \varsigma_{i k \pm l m}=\varsigma_{i k} \pm \varsigma_{l m} .
$$


In the particular case of particle motion orthogonal to the defect plane $\left(p_{1}=0, p_{2}=0, \kappa=\omega\right)$, the amplitudes of reflected and transmitted particles are given by

$$
\begin{aligned}
& b_{-}^{1}=\frac{c_{-}^{2}\left(\omega\left(\omega \varsigma_{14-23}-i p_{0} \varsigma_{13+24}\right)+m\left(p_{0} \varsigma_{22-33}+i \varsigma_{12-34} \omega\right)\right)}{\left(\left(m^{2}+\omega^{2}\right) \varsigma_{2}^{2}+\left(\omega \varsigma_{1}-i m \varsigma_{3}\right)^{2}\right)}, \\
& b_{-}^{2}=\frac{c_{-}^{1}\left(\omega\left(\omega \varsigma_{14-23}+i p_{0} \varsigma_{13+24}\right)+m\left(p_{0} \varsigma_{33-22}+i \omega \varsigma_{12-34}\right)\right)}{\left(\left(m^{2}+\omega^{2}\right) \varsigma_{2}^{2}+\left(\omega \varsigma_{1}-i m \varsigma_{3}\right)^{2}\right)}, \\
& c_{+}^{1}=\frac{c_{-}^{1} \omega\left(\omega \varsigma_{1}+i p_{0} \varsigma_{2}-i m \varsigma_{3}\right)}{\left(\left(m^{2}+\omega^{2}\right) \varsigma_{2}^{2}+\left(\omega \varsigma_{1}-i m \varsigma_{3}\right)^{2}\right)}, \quad c_{+}^{2}=\frac{c_{-}^{2} \omega\left(\omega \varsigma_{1}-i p_{0} \varsigma_{2}-i m \varsigma_{3}\right)}{\left(\left(m^{2}+\omega^{2}\right) \varsigma_{2}^{2}+\left(\omega \varsigma_{1}-i m \varsigma_{3}\right)^{2}\right)} \text {. }
\end{aligned}
$$

\section{Bound states}

States localized in the vicinity of $x_{3}=0$ arise if $\bar{p}^{2}-m^{2}<0$ and $\kappa(\bar{p})=i|\kappa(\bar{p})|$ is imaginary. For them by $p_{0}>m$, the dispersion relation $p_{0}^{2}-p_{1}^{2}-p_{2}^{2}-\xi^{ \pm} m^{2}=0$ is satisfied with

$$
\xi^{ \pm}=1-\left(\frac{\varsigma_{1} \varsigma_{3} \pm \sqrt{\varsigma_{2}^{2}\left(1+\varsigma_{4}^{2}\right)}}{\varsigma_{1}^{2}+\varsigma_{2}^{2}}\right)^{2} .
$$

By fixed parameters $\varsigma_{i}, i=1 \ldots 4$, there are two possible values for corresponding normalized electric current $\vec{J}=e \bar{\psi} \vec{\gamma} \psi / \bar{\psi} \psi$, which read

$$
\vec{J}\left(x_{3}, \bar{p}\right)=\exp \left\{-\sqrt{\left(1-\xi^{ \pm}\right)^{2}} m\left|x_{3}\right|\right\} \vec{j}(\bar{p}), \vec{j}(\bar{p})=\frac{e \bar{\chi}_{+}(\bar{p}) \vec{\gamma} \chi_{+}(\bar{p})}{\bar{\chi}_{+}(\bar{p}) \chi_{+}(\bar{p})}=\frac{e \bar{\chi}-(\bar{p}) \vec{\gamma} \chi_{-}(\bar{p})}{\overline{\chi_{-}}(\bar{p}) \chi_{-}(\bar{p})},
$$

where $e$ is a charge of the particle. Here, $j_{3}(\bar{p})=0$, and the parallel to the plane $x_{3}=0$ components of $\vec{j}(\bar{p})$ can be written as follows

$$
j_{1}(\bar{p})=-\frac{e p_{1}}{m \xi^{ \pm}}, \quad j_{2}(\bar{p})=-\frac{e p_{2}}{m \xi^{ \pm}} .
$$

The coefficient $\xi^{ \pm}$at the corresponding values of the parameters $\varsigma_{i}$ can be positive or zero. For example, $\xi^{+}=0$ at $\varsigma_{1}=\varsigma_{3}, \varsigma_{2}=1, \varsigma_{4}=0$, and $\xi^{-}=0$ at $\varsigma_{1}=-\varsigma_{3}, \varsigma_{2}=1, \varsigma_{4}=0$. If $\varsigma_{2}=0$, $\left|\varsigma_{1}\right|>\sqrt{1+\varsigma_{3}^{2}}$, and $\varsigma_{4}= \pm \sqrt{\varsigma_{1}^{2}-1-\varsigma_{3}^{2}}$, then $\xi^{ \pm}>0$.

The dispersion law $\bar{p}^{2}=\xi^{ \pm} m^{2}$ describes free particles with the effective mass $m \sqrt{\xi^{ \pm}}<m$ in the $(2+1)$ - dimensional space-time with two spatial coordinates and one temporal coordinate if $\xi^{ \pm}>0$. If $\xi^{+}=0$ or $\xi^{-}=0$, then the corresponding particles are massless. The motion of such particles explains numerous effects in graphene.

\section{Conclusions}

We have constructed a model of the interaction of a spinor field with a material plane in the framework of Symanzik's approach. The action functional of the model includes the standard spinor Dirac action in $(3+1)$ dimensional space-time and an additional contribution from the defect, concentrated in the part of space that it occupies (in considered case it was the plane $x_{3}=0$ ). The action contains three parameters characterizing the properties of the material of the plane. In the model, we calculated the characteristics of a Dirac particle scattering on the defect plane and also studied the properties of states localized near it. 
The model and the obtained results can be used to describe theoretically processes of the interaction of electrons, with two-dimensional materials (graphene, thin films, sputter, sharp boundaries of a solid body). Simple modifications of the model allow taking the effects of the action of external electromagnetic fields into account.

Applying Symanzik's approach to QED enables constructing an universal model for a wide class of quantum macroscopic phenomena in two-dimensional materials. These models can prove useful in investigating the Hall effect, plasmon-polariton effects, nanophotonics, properties of topological insulators, thin films, sputters, and sharp boundaries [15].

Acknowledgments. This paper was supported in part by St. Petersburg State University (Grant No. 11.38.660.2013).

\section{References}

[1] N. N. Bogoliubov and D. V. Shirkov, Quantum Fields, Nauka, Moscow (1993); C. Itzykson and J.-B. Zuber Quantum Fields Theory, McGraw-Hill, New York (1980).

[2] H. B. G Casimir, Proc. K. Ned. Akad. Wet B. 51, 793-795 (1948).

[3] U. Mohideen and A. Roy, Phys. Rev. Lett. 81, 4549-4552 (1998); arXiv:physics/9805038v2 (1998);

A. Roy, C.-Y. Lin and U. Mohideen, Phys. Rev. D 60, 111101(R) (1999); arXiv:quantph/9906062v3 (1999).

[4] B. W. Harris, F. Chen and U. Mohideen, Phys. Rev. A 62, 052109 (2000); arXiv:quantph/0005088v1 (2000).

[5] G. Bressi, G. Carugno, R. Onofrio and G. Ruoso, Phys. Rev. Lett. 88, 041804 (2002); arXiv:quant-ph/ 0203002v1 (2002).

[6] M. Bordag, G. L. Klimchitskaya, U. Mohideen, and V. M. Mostepanenko Advances in the Casimir Effect, (Intl. Ser. Monogr. Phys., Vol. 145), Oxford Univ. Press, Oxford (2009); G. L. Klimchitskaya, U. Mohideen and V. M. Mostepanenko, Rev. Mod. Phys. 81, 1827-1885 (2009); arXiv:0902.4022v1 [cond-mat.other] (2009).

[7] K. A. Milton, J. Phys. A: Math. Gen. 37, R209-R277 (2004).

[8] K. Symanzik, Nucl.Phys. B 190, 1-44 (1981). .

[9] V. N. Markov and Yu. M. Pis'mak, J. Phys. A: Math. Gen. 39, 6525-6532 (2006); arXiv: hepth/0505218v3 (2005).

[10] V. N. Marachevsky and Yu. M. Pis'mak, Phys. Rev. D 81, 065005 (2010); arXiv:0907.1985v2 [hep-th] (2009).

[11] D.Yu. Pis'mak and Yu. M. Pis'mak, Theor. Math. Phys. 175,816-826 (2013); Phys. Part. Nuclei 44, 450-461 (2013); Theor. Math. Phys. 166, 1423-1431 (2011).

[12] D.Yu. Pis'mak, Yu. M. Pis'mak and F. J. Wegner, Phys. Rev. E 92, 013204 (2015); arXiv:1406.1598v1 [hep-th] (2014).

[13] I. V. Fialkovsky, V. N. Markov and Yu. M. Pis'mak, J. Phys. A: Math. Gen. 39, 6357-6363 (2006); Int. J. Mod. Phys. A 21, 2601-2616 (2006); arXiv:hep-th/0311236v2 (2003).

[14] D. Yu. Pismak, Yu. M. Pismak, Theor. Math. Phys. 184, 3, 1329-1341 (2015).

[15] G. Bracco and B. Holst (Eds.), Surface Science Techniques, (Springer Ser. Surf. Sci., Vol. 51), Springer, Berlin (2013); M. L. Brongersma and P. G. Kik (Eds.), Surface Plasmon Nanophotonics, (Springer Ser. Opt. Sci., Vol. 131), Springer, Berlin (2007); S. A. Maier Plasmonics: Fundamental and Applications, Springer, Berlin (2007). 